\title{
Perspective
}

PERSPECTIVE Actualité en histoire de l'art

$2 \mid 2020$

Danser

\section{Scandaleuses, marginalisées et hors-la-loi : mes notes de terrain sur les archives (vivantes) de l'histoire de la danse iranienne}

Infamous, Marginal, and Outlawed: My Fieldnotes to the (Living) Archives of Iranian Dance History

Unverschämt, unerheblich, verboten: Aufzeichnungen aus meiner Feldforschung $z u$ den (lebenden) Archiven der iranischen Tanzgeschichte

Infame, Marginale e Fuorilegge: i miei appunti sul campo riguardo gli archivi (viventi) della storia della danza iraniana

Infames, marginales e ilegales: mis notas de terreno alrededor de los archivos (vivos) de la historia de la danza iraní

\section{Ida Meftahi}

Traducteur : Françoise Jaouën

\section{(2) OpenEdition}

\section{Journals}

\section{Édition électronique}

URL : https://journals.openedition.org/perspective/21477

DOI : $10.4000 /$ perspective. 21477

ISSN : 2269-7721

\section{Éditeur}

Institut national d'histoire de l'art

\section{Édition imprimée}

Date de publication : 30 décembre 2020

Pagination : 237-248

ISBN : 978-2-917902-90-5

ISSN : $1777-7852$

\section{Référence électronique}

Ida Meftahi, «Scandaleuses, marginalisées et hors-la-loi : mes notes de terrain sur les archives (vivantes) de l'histoire de la danse iranienne », Perspective [En ligne], 2 | 2020, mis en ligne le 30 juin 2021, consulté le 30 juillet 2022. URL : http://journals.openedition.org/perspective/21477 ; DOI : https://doi.org/10.4000/perspective.21477 


\title{
Scandaleuses, marginalisées et hors-la-loi : mes notes de terrain sur les archives (vivantes) de I'histoire de la danse iranienne
}

\author{
Ida Meftahi
}

Cet essai fournit quelques points de repère sur les origines de mon livre, Gender and Dance in Iran: Biopolics on Stage (Londres, Routledge, 2016). Premier ouvrage historiographique consacré à la danse dans l'Iran du $\mathrm{XX}^{\mathrm{e}}$ siècle, il se concentre sur la transformation du corps dansant sur scène, son espace de performance et l'idéologie culturelle du regard qui l'accompagne. Il examine le corps dansant dans divers milieux sur une période d'un siècle, parmi lesquels la prestigieuse scène de l'art national (1920-1979), l'espace populaire des cafés et des cabarets, les théâtres commerciaux de la rue Lalehzar (quartier historique des loisirs nocturnes de Téhéran) et le cinéma commercial de l'époque précédant la révolution (1930-1979), ainsi que la scène théâtrale islamisée de la période postrévolutionnaire (de 1979 à nos jours). Quatre ans après la parution du livre, le contexte historique particulier qui avait présidé à son élaboration justifie que j'explique certains aspects du processus de recherche, de mes expériences et de mes observations. Depuis la révolution de 1979, le terme " danse " avait en effet disparu du discours officiel en Iran.

Pratiquant moi-même la danse, et ayant grandi en Iran, je suis la première universitaire formée en Occident à mener, de l'intérieur, une étude de terrain sur la danse iranienne. J'avais donc un double lien - émique et étique - avec ce qui était alors la communauté semi-clandestine de la danse, ce qui m'a permis, par diverses voies, de recueillir des données à la fois dans les archives et grâce aux méthodes ethnographiques. Je me suis cependant retrouvée dans une impasse lorsque j'ai commencé à m’intéresser à la danseuse de cabaret dans la période prérévolutionnaire. Des décennies après avoir été exclues de la sphère publique, car elles avaient été considérées comme les symboles même de la corruption sociale et de la nudité sous l'ancien régime, mes interlocutrices vieillissantes souffraient encore de la honte, des stéréotypes négatifs et des mythes sociaux associés à leur passé. Je ne suis parvenue à réinscrire dans l'histoire ce groupe subalterne à la réputation controversée qu'avec l'aide de ces courageuses anciennes danseuses de cabaret, qui ont généreusement accepté de me raconter leur quotidien avant la révolution. 


\section{Professionnelle et clandestine ? \\ Formation et spectacles de danse dans l'Iran postrévolutionnaire}

C'est dans les années 1980, à l'adolescence, que j'ai découvert la communauté clandestine de la danse en Iran, lorsque ma mère Surayya, toujours soucieuse de mon bien-être, s'est brusquement dit que l'apprentissage de la danse m'aiderait à prendre confiance en moi. Cette communauté allait devenir mon principal sujet d'étude. Cependant, contrairement à bien des mères aimantes à travers le monde, la mienne était confrontée à un choix limité de cours appropriés. C'était principalement dû aux mesures restrictives instaurées dans les premières années de la République islamique afin de restaurer la décence des mœurs et des tenues de ses citoyens, mais aussi à l'austérité imposée par la crise provoquée par la guerre Iran-Iraq (1980-1988). De bouche-à-oreille, ma mère a fini par trouver un couple arménien âgé qui dirigeait un studio de danse à Téhéran depuis les années 1950. N’étant pas musulmans, les membres de la communauté arménienne n'étaient pas soumis au même degré de surveillance par l'État islamique (fig. 1). Lazarian et son mari s'étaient reconvertis aux cours d'aérobic réservés aux femmes, tout en enseignant, non officiellement, divers types de danses caucasiennes et classiques, ainsi que des danses de couple dont la valse, le tango et le cha-cha-cha ${ }^{1}$.

Mon avenir en tant que danseuse professionnelle, puis chercheuse en danse, fut enfin scellé lorsque, après des années de tentatives infructueuses, ma mère parvint à m'inscrire dans le cours de Farzaneh Kaboli, danseuse étoile de l'Organisation nationale folklorique (1967-1979), qui jouissait d'un grand prestige avant la révolution. Il s'agissait d'une troupe bénéficiant d'un financement public qui, à l'instar de la Compagnie iranienne de ballet (1956-1979), avait été créée dans le but d'adapter pour la scène le folklore, la mythologie et la littérature classiques iraniens, et de reconfigurer l'esthétique iranienne de la danse en y intégrant des formes et une pédagogie occidentales, en s'efforçant de donner ses lettres de noblesse à cet $\operatorname{art}^{2}$.

1. Farzaneh Kaboli, Organisation nationale folklorique, programme, 1972, archives personnelles d'Ida Meftahi.

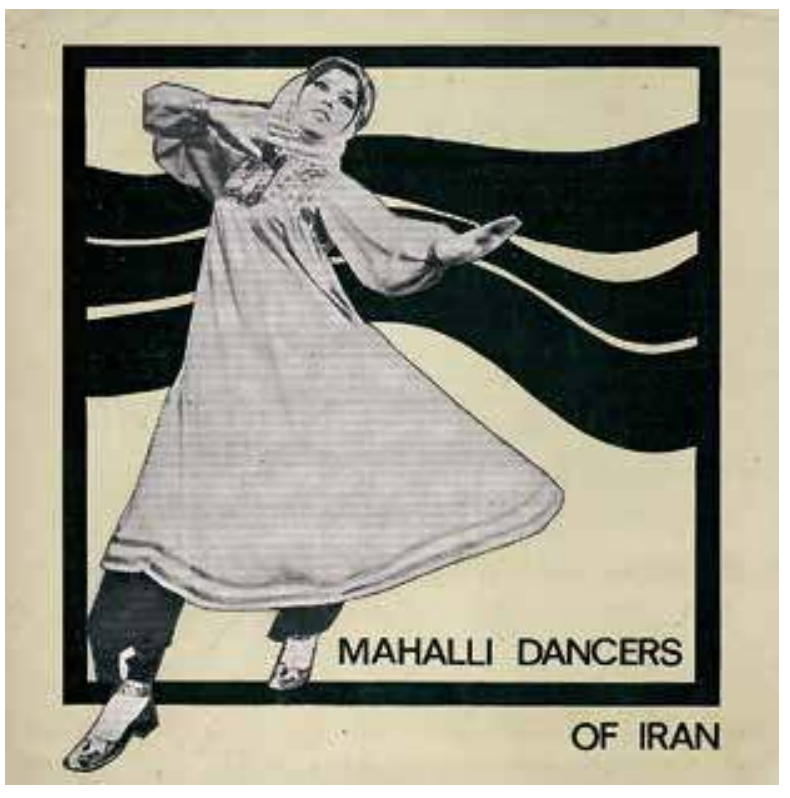

Membre permanent de la compagnie, Kaboli s'était produite dans des spectacles de danse folklorique ou nationale traditionnelle dans des cadres prestigieux, artistiques ou diplomatiques, à la fois en Iran et à l'étranger.

Après la révolution, l'interdiction visant la danse eut un impact désastreux sur la carrière de Kaboli, puisque sa troupe avait été dissoute. Employée du ministère des Arts et de la Culture, elle survécut en se reconvertissant officiellement en actrice de théâtre, de cinéma et de télévision. C'est l'émission télévisée Little Ali, où elle jouait la mère d'Ali, qui fit d'elle une icône pour les enfants ayant grandi, comme moi, pendant la période de la guerre. Je faisais partie des nombreux spectateurs de ce feuilleton lent, à petit budget, dont l'intrigue sombre et l'esthétique minimaliste d'avant-garde ne m'attiraient pas particulièrement. Mais la chanson entraînante du générique et le 


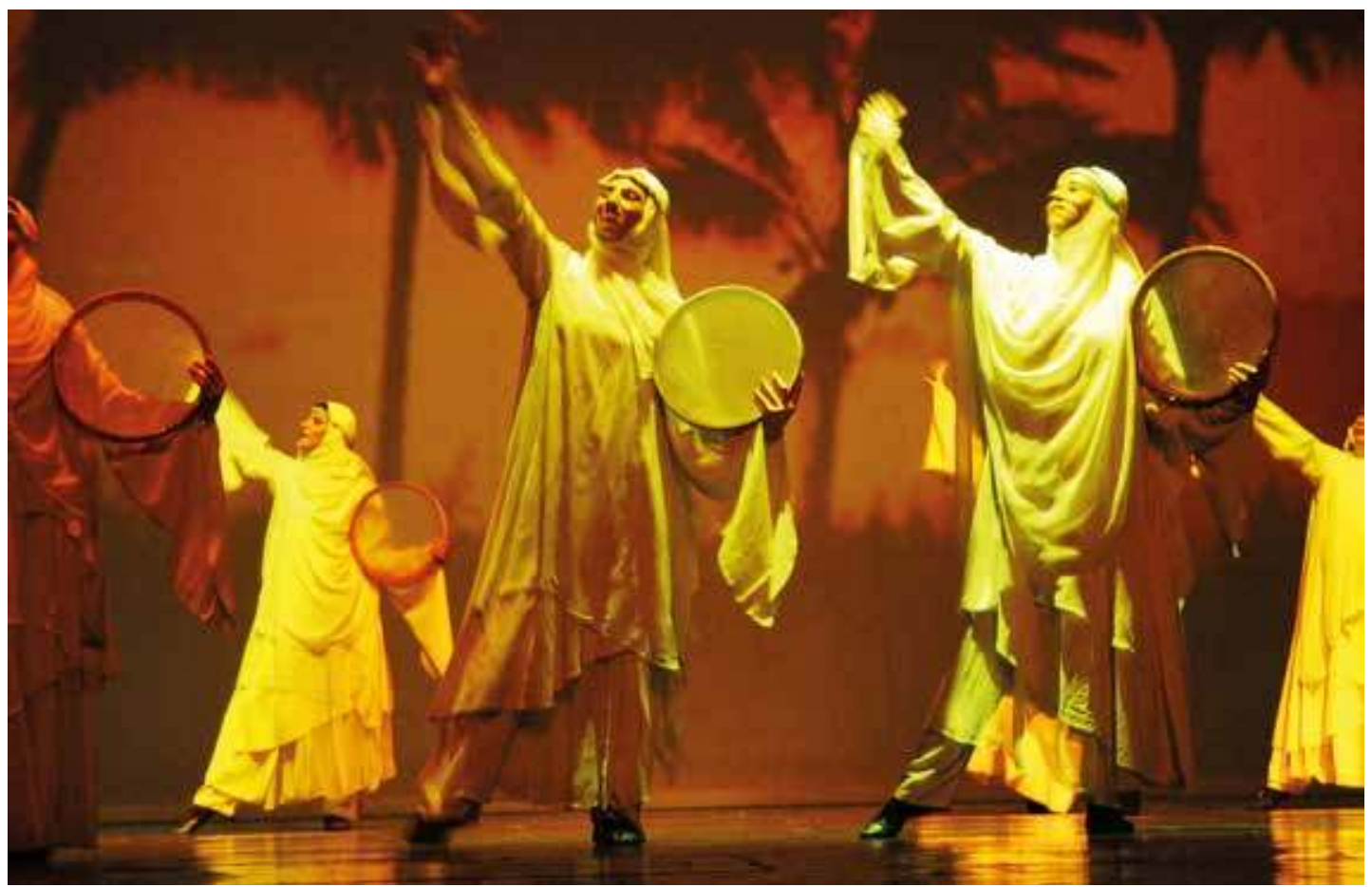

visage innocent d'Ali, dont le père était parti à la guerre, avaient fait du personnage de Kaboli un symbole pour 2. Hamasah'i inqilab-i sang [The Epic of the Rock Revolution], quantité de femmes élevant seules leurs enfants. 2000. Photographie Omid Salehi.

$\mathrm{Au}$ quotidien, " la mère du petit Ali " poursuivait en coulisses son ancienne carrière en donnant chez elle des cours de danse privés à des femmes et à des jeunes filles.

Très rapidement, les amoureux de la danse à Téhéran rêvèrent de s'inscrire aux cours prestigieux et relativement coûteux qu'elle offrait. Entre-temps, elle saisissait toutes les occasions de ramener la danse sur la scène publique. Au théâtre, elle repoussait les limites de cet art en y intégrant des segments fondés sur le mouvement et, en privé, elle formait un ensemble d'élèves qui seraient capables de monter sur un plateau à la première opportunité. Son vœu fut exaucé en 1998, lorsqu'une organisation caritative lui demanda de créer des spectacles musicaux réservés à un public féminin dans le gymnase de l'École italienne de Téhéran (qui bénéficiait de l'immunité diplomatique). Je faisais partie des quelques danseuses qui eurent le privilège de se produire à cette occasion (ainsi que les deux années suivantes), et à découvrir par moi-même l'esprit de persévérance et de pratique, la passion et l'enthousiasme pour cet $\operatorname{art}^{3}$. C'est à ce moment-là que j'ai songé à écrire un ouvrage de recherche sur la danse en Iran, un projet qui, pour la plupart de mes collègues, paraissait irréaliste.

Au moment où j'entamais, au Canada, une maîtrise sur l'histoire de la danse dans l'Iran prérévolutionnaire, mes collègues se produisaient au théâtre dans des spectacles de danse officiels du genre harikat-i-mawzun ( mouvements rythmiques" sur un sujet chaste), parfois au côté de danseurs masculins, ce qui ne s'était pas vu depuis la révolution ${ }^{4}$ (fig. 2).

Ce miracle de la scène iranienne (bien que placé sous le signe de la chasteté) résultait d'un long processus de négociation entre artistes déterminés, sensibilités islamiques en évolution et politique culturelle officielle. En tant qu'anciens employés d'institutions 


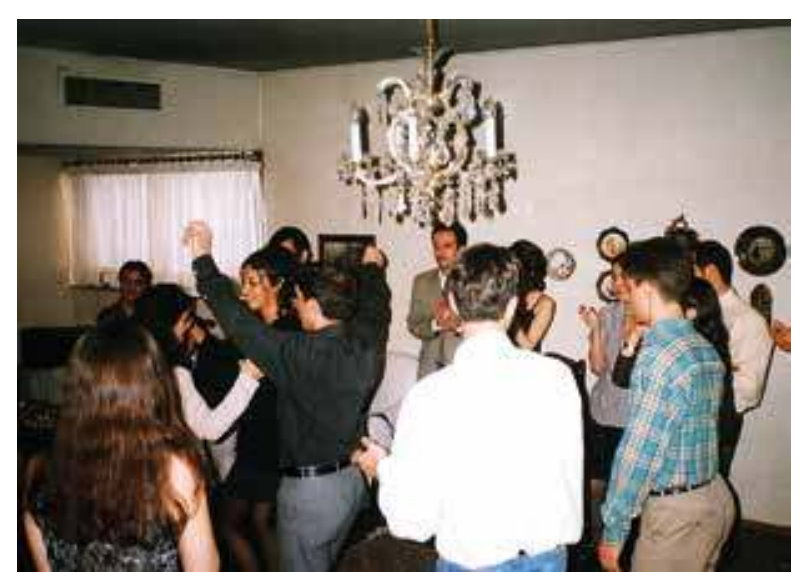

3. Danse sociale lors d'une réunion privée, Téhéran, 1999, archives personnelles d'Ida Meftahi. gouvernementales, Kaboli et ses collègues masculins qui avaient survécu dans le système théâtral pendant la période postrévolutionnaire se joignirent à l'entreprise. Après tout, Kaboli s'était engagée à " rester " jusqu'à ce qu'elle puisse "danser à nouveau sur la scène de la salle Roudaki ${ }^{5}$ ". Elle avait choisi pour slogan « être aussi solide qu'un clou, qui devient plus solide à mesure qu'on le frappe ! "

Le climat culturel plus détendu des années 2000 et la présence insistante de corps dansants dans les émissions de chaînes de télé satellites, diffusées depuis les quatre coins de la planète, ont donné une nouvelle impulsion à divers styles de danse qui ont attiré des élèves enthousiastes de tous âges. Occupant l'espace liminal situé entre public et privé, entre officiel et semi-clandestin, les

salles de sport et les jardins d'enfants ajoutèrent eux aussi la danse à leurs programmes. Ce glissement de la danse vers le sport a permis à divers genres de trouver un espace officiel dans l'éducation physique en Iran.

Il est toujours officiellement interdit de danser en public en Iran, et le simple observateur pourrait trouver contre-productif cet engouement pour l'apprentissage de la danse. Mais le phénomène s'appuie sur une tradition profondément enracinée de " danse sociale " qui pouvait transformer un rassemblement festif privé en une occasion de danse collective (fig. 3). Depuis les années 1990, les réjouissances liées à des événements sportifs ou politiques ont parfois donné lieu à des situations carnavalesques, dont ont profité les hommes et les femmes pour laisser libre cours à cet élan clandestin en se mettant hardiment à danser dans les rues ${ }^{6}$.

Au cours des dernières années, la danse mixte en public s'est créée un espace dans les parcs, où les municipalités ont mis en place des programmes d'aérobic auxquels participent souvent des personnes âgées. La danse sur scène a également trouvé, sous de multiples formes, différents lieux d'expression. C'est notamment le cas des harikat-i-mawzun, que l'on peut voir à l'occasion de spectacles religieux, épiques ou musicaux, mais aussi lors de concerts où ils accompagnent de la musique traditionnelle ou pop, durant des festivals folkloriques officiels et des concerts donnés l'après-midi devant un public exclusivement féminin avec la sanction des autorités, ou encore pendant des spectacles d'avant-garde se déroulant dans des espaces minimalistes ${ }^{7}$.

\section{Conventions sociales et danse dans la sphère privée}

La prédominance de la danse dans la vie privée des Iraniens ne signifie pas pour autant qu'il n'existe dans ce cadre aucune convention sur la pratique, qu'il s'agisse d'y participer ou de la regarder. La célèbre anthropologue iranienne Soheila Shahshahani a ainsi montré que l'âge et le genre des participants jouent un rôle important s'agissant de la danse sociale dans la sphère privée, tout comme le degré de formalité ou d'intimité entre 
individus ${ }^{8}$. Par ailleurs, occuper la position de simple spectateur lorsqu'on est soi-même incapable de danser peut créer un sentiment d'aliénation chez ceux qui ont été élevés dans un environnement familial " sans danse " .

Le simple observateur serait tenté de faire un lien entre ces conventions concernant la vie privée et celles imposées par la République islamique dans la sphère publique. Mais Anthony Shay, qui a mené des recherches inédites sur la diaspora iranienne, a montré que ces traits ont survécu à la révolution ${ }^{10}$. Auteur du premier ouvrage universitaire écrit sur la danse iranienne selon les critères académiques aujourd'hui en vigueur, Shay a inventé le terme choreophobia pour désigner la passion effrénée des Iraniens pour la danse, en dépit des effets délétères et paralysants des normes socioculturelles, des codes de moralité et des stigmates attachés à cette discipline.

\section{" Dépravées " et subalternes : les danseuses (de cabaret) comme sujets de l'histoire orale dans les années 2010}

Au cours des années de travail de terrain, ces complexités sociales et politiques concernant la danse ont dirigé mes recherches, tout comme elles dirigeaient le quotidien de mes sujets vivants ou disparus. Malgré ces difficultés, et grâce à mon approche holistique de la danse, j'ai pu m'entretenir avec des douzaines d'artistes - acteurs de théâtre, musiciens, danseurs - et avec des chercheurs de formation, de milieux et d'âges différents, de notoriété plus ou moins grande en Iran et à l'étranger. Mes liens avec la communauté harikat-i-mawzun et avec les artistes appartenant à la " haute culture " du ballet et de la danse nationale m'ont permis de rencontrer et d'interviewer deux générations de danseuses iraniennes de l'époque prérévolutionnaire. Même si la révolution de 1979 a mis fin à leur carrière, leur prestigieux passé artistique fait aujourd'hui encore leur fierté, comme elles en ont souvent témoigné au cours de nos conversations.

J'ai en revanche eu beaucoup de difficultés à retrouver d'anciennes danseuses de cabaret, dont l'esthétique et le " prestige " ne correspondaient guère au style pratiqué par mon réseau de contacts. Trois décennies après la révolution de 1979 qui incendia les cabarets, ainsi que les maisons de passe et les cinémas, les danseuses de cabaret n'étaient plus associées dans la mémoire collective qu'aux « tenues semi-dénudées » et aux « mouvements érotiques ", image heurtant les sensibilités d'un public islamisé, jurant avec l'apparition de femmes voilées en public. Comme je l'ai montré dans mon livre, les mesures radicales prises après la révolution répondaient au désir de purger la société de tous les signes de corruption. Produit dérivé de trois discours parallèles - nationaliste, gauchiste anti-impérialiste, et islamique anticolonialiste et anti-obscénité -, cette volonté d'éliminer le sexuel pour le bien de la société était déjà en germe des décennies avant la révolution ${ }^{11}$. En outre, les positions critiques adoptées par les représentants de la gauche artistique ou des arts nationalistes contre toutes les formes de divertissement urbain, sous le règne des Pahlavi, avaient marqué ces femmes du sceau de la corruption, de la dégénérescence et de la médiocrité intellectuelle. Ces notions de nature idéologique ont eu un effet durable sur les arts en imposant un goût esthétique élitiste après la révolution ${ }^{12}$.

On peut se demander comment les mythes et les stéréotypes en vigueur avant la chute du shah ont survécu au temps et continuent à hanter l'existence, la mémoire et les familles de ces femmes des décennies après leur effacement brutal de l'espace public. Cette persistance est en partie due à la popularité durable du cinéma commercial prérévolutionnaire sur le marché noir postrévolutionnaire (et désormais sur YouTube 
et les réseaux satellites persans diffusant depuis l'étranger). Les passionnés de danse de cabaret peuvent ainsi avoir accès, non seulement à des longs métrages, mais également à des compilations de scènes filmées montrant leurs danseuses préférées ; ils peuvent aussi se procurer d'excellentes photographies des artistes en train de danser, à partir des négatifs conservés par de vieux studios photo à travers le pays (fig. 4). À l'époque, ces photos étaient affichées à l'entrée des cinémas, des théâtres et des cabarets, ou étaient publiées dans la presse pour attirer les spectateurs ou les lecteurs.

Après avoir pris contact avec des amis travaillant dans le milieu du théâtre et du cinéma, et avec mon marchand habituel d'objets liés aux médias, un homme d'apparence pieuse qui m'a confié les coordonnées d'anciennes danseuses de cabaret et actrices de cinéma, j'ai découvert que nombre d'artistes connues avaient quitté le pays, et que les occasions d'interview seraient rares. De surcroît, même lorsque j'ai réussi à retrouver une ancienne danseuse de cabaret, il m'a fallu gagner sa confiance pour qu'elle accepte de prendre le risque d'évoquer sa carrière d'avant la révolution. J'ai dû faire jouer mes relations pour parvenir à contacter deux actrices de cinéma de l'époque, qui avaient toutes deux incarné des danseuses de cabaret à l'écran. L'une d'elles a refusé de me recevoir, et le mari de l'autre m'a interdit de parler à son épouse, par crainte du scandale. J'ai finalement réussi à convaincre le regretté Murtiza Ahmadi, le très célèbre acteur de spectacles de musique populaire, de théâtre et de cinéma, de me raconter ses souvenirs vieux de plusieurs décennies. Il n'a pu me fournir qu'un aperçu de l'existence des femmes employées dans le milieu du divertissement urbain, en me mettant en garde contre leur image excessivement négative dans l'imaginaire socio-historique.

\section{Mon sésame pour accéder à l'univers étrange de l'industrie du divertissement sous le règne des Pahlavi}

J'ai enfin atteint mon but lorsque, grâce à un acteur de théâtre de ma connaissance, j'ai été présentée à une ancienne actrice d'une soixantaine d'années prénommée Rahilah, qui s'était autrefois produite dans les théâtres de la rue Lahlezar et qui, m'avait-on dit, avait également été danseuse de cabaret avant la révolution. Elle faisait partie des quelques artistes relativement méconnues liés au théâtre musical du quartier qui avaient réussi à poursuivre une carrière théâtrale après la révolution. Pour la plupart des anciennes danseuses de cabaret et leurs familles, la stratégie de survie a été d'enterrer le passé et d'effacer toute trace de leur ancienne activité, désormais source de honte.

Faisant confiance à mon contact, et donc à moi-même (surtout lorsque j'ai évoqué mon passé de danseuse), Rahilah semblait toute disposée à me parler de son expérience du cabaret. Pendant notre entretien, qui dura plusieurs heures, elle me raconta l'univers de l'industrie du divertissement de l'ère des Pahlavi. J'ai suivi pas à pas l'histoire de sa vie à mesure qu'elle décrivait les sphères, apparemment distinctes, mais en réalité étroitement liées, de la culture urbaine du divertissement avant la révolution. Elle me parla des mutribi (troupes de divertissement traditionnel se produisant lors de mariages ou d'autres événements) ${ }^{13}$, des théâtres commerciaux, du cinéma commercial baptisé film-i-farsi, ainsi que des cafés musicaux et des cabarets. Malgré la réputation morale et esthétique médiocre de tous ces genres, Rahilah était fière de sa carrière d'artiste dans le quartier Lalehzar qui, selon elle, avait donné naissance au théâtre moderne iranien. La danse était le seul moyen pour elle de gagner sa vie et de subvenir aux besoins de sa fille et de sa mère âgée. 
Elle commença tout d'abord par incarner une danseuse de cabaret dans les attraksiun du théâtre où elle était devenue actrice. Ces interludes courts de pas de danse, de numéros d'acrobatie ou de jonglerie, qui intervenaient entre les principales sections des pièces jouées, visaient à attirer un plus large public. Rahilah me raconta que ses collègues masculins avaient été scandalisés de la voir prendre ce nouvel emploi, où elle dansait en tenue légère. Ils allèrent même jusqu'à exiger du directeur de leur troupe qu'il augmente son salaire d'actrice afin qu'elle ne soit pas obligée de danser.

Mais les salaires offerts par l'industrie du divertissement nocturne dans le Téhéran des années 1960 étaient trop attractifs pour empêcher Rahilah de se produire dans les cabarets et d'accompagner ses collègues des mutribi aux mariages et autres réjouissances familiales. Toutefois, cette carrière prospère lui coûta sa famille, qui brisa définitivement tout lien avec elle, un sort souvent réservé aux femmes artistes de l'ère des Pahlavi. Selon Rahilah, ce geste radical de la part de sa famille s'explique par le stigmate social attaché aux femmes se produisant en public, notamment dans les cafés et les cabarets, souvent considérés comme des lieux où régnaient la vulgarité, la prostitution, l'alcoolisme voire la violence.

Elle me raconta les dangers auxquels elle était confrontée sur scène, l'indiscipline du public et les violentes altercations à la fin du spectacle, ce qui l'avait contrainte à se procurer une arme blanche. Ces bagarres à la porte du théâtre étaient souvent provoquées par des hommes se querellant pour obtenir un rendez-vous avec les danseuses. Celles-ci devaient se plier à cette tâche en raison d'une pratique illégale et mal connue, mais très répandue dans le milieu de la nuit, baptisée fishkhuri, selon laquelle les clients achetaient des jetons pour boire un verre avec leurs artistes préférées. Les danseuses étaient donc confrontées à la sortie à de multiples prétendants à leur compagnie exclusive pour la soirée. Malgré les risques et les handicaps sociaux associés à l'industrie "florissante " de la nuit, les avantages financiers d'une carrière de danseuse attiraient même les femmes mariées des quatre coins du pays.

Comme beaucoup d'autres, Rahilah était forcée de mener une double vie en raison des stigmates attachés à la danse, ce qui semble avoir lourdement pesé sur son état d'esprit au fil des ans. Pendant notre entretien, elle paraissait basculer d'une personnalité à l'autre selon le sujet abordé. Elle gardait beaucoup de bons souvenirs de sa vie de danseuse, se lançant dans des improvisations comiques sur un ton gouailleur ${ }^{14}$. Elle se rappelait avec plaisir l'esprit d'amitié et de camaraderie qui régnait entre ses collègues de cabaret ; en coulisses, elles apprenaient et répétaient les pas des dernières danses populaires à la mode ou vues au cinéma ;

4. La danseuse de cabaret Nadiya, dans les années 1970, archives personnelles d'Ida Meftahi.

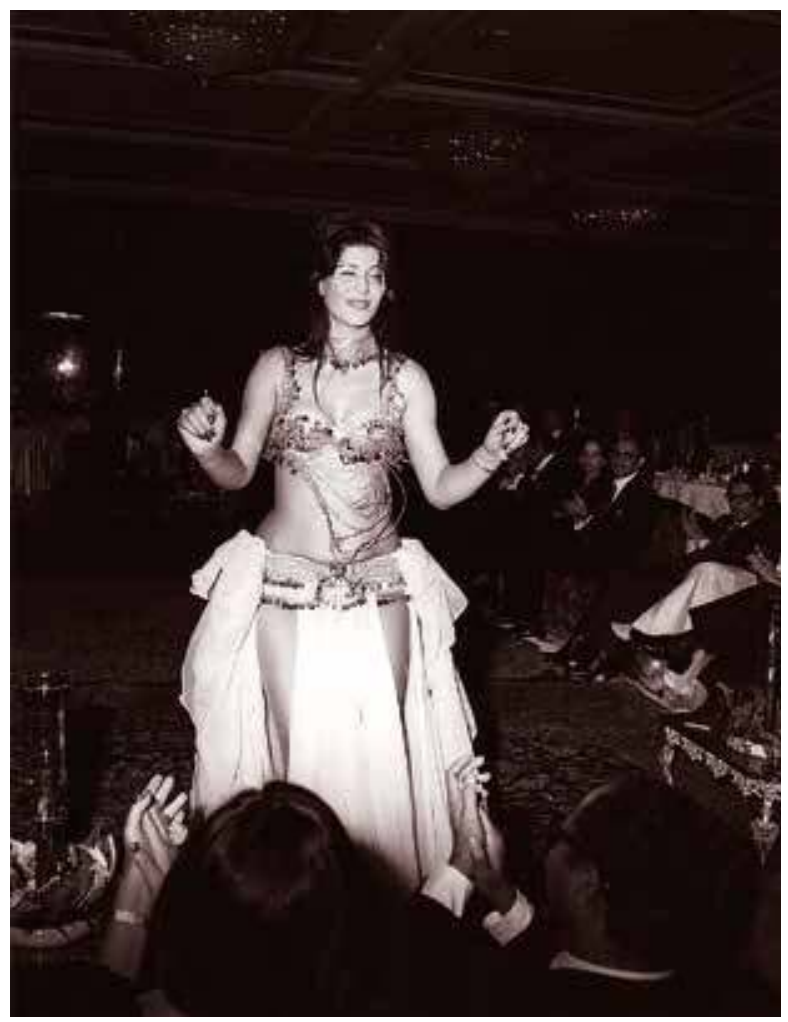




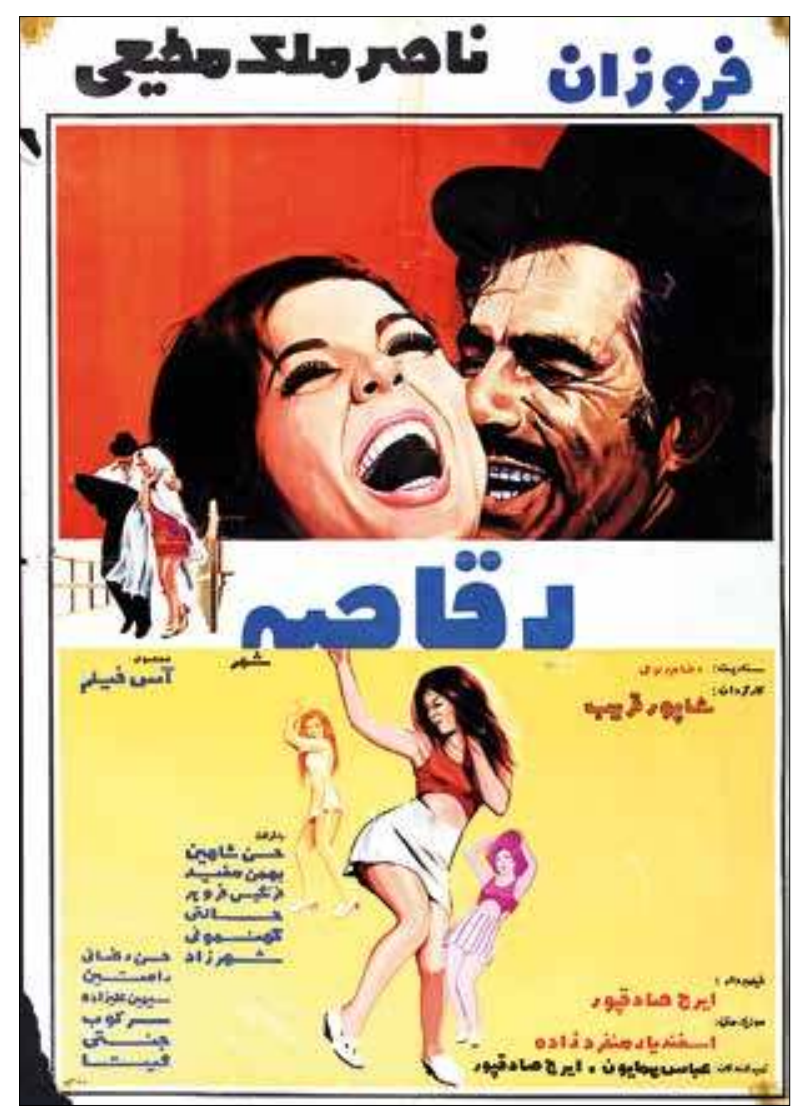

5. Raqqasah'i Shahr [City's dancer], 1970, affiche de film, archives personnelles d'Ida Meftahi. hors de la scène, elles s'entraidaient pour prendre soin de leurs jeunes enfants et participaient même à des activités religieuses, notamment la collecte de nourriture pour les pauvres appelée nazri pukhtan, organisée lors des fêtes musulmanes. Sentant ma confusion devant ces signes de religiosité chez des femmes travaillant dans le milieu très " non-islamique " des cabarets, Rahilah me confirma qu'elle avait été élevée dans le respect de la religion, et que nombre de femmes travaillant dans le milieu de la nuit étaient pratiquantes.

Il était d'ailleurs impossible de ne pas remarquer que Rahilah était animée d'une foi profonde qui la fit changer de discours à plusieurs reprises, passant de la comiquedanseuse à la femme pieuse rendant grâces au Tout-puissant, au Prophète et à d'autres figures saintes du shiisme. Notre conversation fut également interrompue par des appels téléphoniques de parents inquiets des répercussions que pourrait avoir cette interview. (En raison de ces complications socioculturelles et familiales, j'ai choisi d'utiliser des pseudonymes dans toutes mes publications afin de protéger mes interlocutrices.) Malgré tout, Rahilah était suffisamment motivée pour me faire rencontrer Naghmah, une vieille amie et ancienne danseuse de cabaret âgée de près de quatre-vingts ans ${ }^{15}$. L'atmosphère douil-

lette et réconfortante du modeste appartement de Naghmah, dans le Sud de Téhéran, permit aux vieilles dames de se détendre suffisamment pour me confier des détails clés sur la longue carrière qu'elles avaient abandonnée trente ans auparavant. Elles m'apprirent notamment qu'elles devaient se soumettre régulièrement à des dépistages de maladies vénériennes afin de garder leur autorisation de travail au cabaret.

\section{Mes recherches pour retrouver des danseuses de cabaret de l'époque prérévolutionnaire en dehors de l'Iran}

Après cet entretien révélateur avec Rahilah et Naghmah, qui m’a permis de mieux comprendre la culture populaire urbaine de l'ère des Pahlavi, j'ai pu rencontrer deux actrices de cinéma et de théâtre de l'époque, Fakhri et Shahin, qui vivaient en maison de retraite à Toronto. Shahin avait connu la célébrité dans les années 1950 et 1960 . Leur ayant présenté mon sujet de recherche (la danse), elles nièrent d'abord toute association passée avec la danse. Malgré la distance créée par plusieurs océans et plusieurs décennies, le terme danse évoquait immédiatement à leurs yeux la danseuse de cabaret 
à la réputation " douteuse ». Elles finirent malgré tout par reconnaître avoir pratiqué divers styles de danse, à la fois au théâtre et au cinéma.

Fakhri et Shahin m'ont mise en contact avec leur amie Ziba, une ancienne actrice septuagénaire qui jouait souvent une danseuse de cabaret au cinéma avant la révolution (on trouve sur YouTube quantité d'extraits de ces films). Soucieuse de distinguer son personnage à l'écran des danseuses de cabaret « à la moralité douteuse », elle m'expliqua que la célébrité apportée par ses rôles lui avait valu la réprobation sociale, notamment dans les régions plus conservatrices. Le cheminement de Ziba vers une notoriété "non-désirée " ressemble à celui de nombreuses artistes de la période. Issue d'une famille juive d'artistes, elle commença à jouer dans une troupe de théâtre comique à l'adolescence. Empêchée de poursuivre sa scolarité, elle apprit seule à lire, une compétence indispensable au travail d'acteur. Elle avait une vingtaine d'années lorsqu'elle divorça de son mari, acteur lui aussi, qui l'avait quittée pour une autre actrice en abandonnant leurs deux petites filles. Elle se lança alors dans une carrière théâtrale dans le quartier Lalehzar de Téhéran. Pour subvenir aux besoins de ses filles, elle dut trouver d'autres emplois, puis finit par décrocher un rôle dans un film, après quoi elle fut régulièrement employée par l'industrie du cinéma commercial pour jouer divers rôles de danseuses de cabaret (fig. 5).

Comme je l'ai montré dans Gender and Dance in Iran, l'apparition récurrente du type de la danseuse de cabaret n'était pas seulement due à l'aspect provocant du personnage ou à la popularité de son répertoire ; elle s'expliquait aussi par le nombre grandissant de danseuses dans le milieu de la nuit et par les mythes sociaux qui les entouraient. Jeune fugueuse ou mère égarée fuyant les mauvais traitements ou la pauvreté, la danseuse de cabaret montrée à l'écran apparaissait sous les traits d'une séduisante femme fatale demandant à être sauvée, domptée et domestiquée par le héros. En cas de transgression, le public masculin assistait alors au châtiment infligé par le héros, qui la giflait en la traitant de « femme perdue ».

La presse populaire reprenait ce genre d'image, profitant de ce personnage, à l'instar du cinéma de l'époque, en donnant un large écho à des rumeurs scandaleuses sur les danseuses et en rattachant symboliquement des mythes sociaux à des artistes populaires existantes ${ }^{16}$. Ces films, qui touchaient un public considérable, exerçaient une pression sociale sur les actrices connues et leur famille, et certaines d'entre elles ont dû renoncer prématurément à leur métier, à l'instar de Ziba. Exilée depuis des décennies, celle-ci était toujours contrainte par sa famille à dissimuler son passé et celui de ses collègues ${ }^{17}$.

Pour procéder à une analyse critique de mes sources orales, je devais tenir compte de l'âge de mes interlocutrices et des réalités quotidiennes imposées par des forces structurelles, mais aussi de leurs préférences, de leurs réticences et de leurs penchants personnels. Elles m'ont toutefois fourni des informations et des indices épars qui m'ont permis de retrouver dans les archives les traces de la danse de cabaret, et de reconstituer l'histoire de nombreuses femmes marginalisées travaillant dans le milieu de la nuit.

\section{La recherche des danseuses de cabaret dans les archives}

À l'instar des recherches sur le terrain, la consultation des archives officielles iraniennes présentait certaines difficultés, allant de la nature " controversée " du sujet à l'absence de toute référence à la danse dans les index et les catalogues, ainsi que les restrictions concernant le visionnage des documents audiovisuels pertinents. J'ai donc mené ma recherche sur les danseuses de la compagnie nationale à partir des documents officiels 
de l'ère des Pahlavi concernant la censure, les règlements sur la culture et la diffusion de la haute culture et des arts nobles, ce qui englobait les troupes de danse financées par le gouvernement.

Les danseuses de cabaret ne bénéficiaient pas d'une sanction officielle, et leur domaine de représentation - cabarets, cinéma, théâtre et musique populaires - n'appartenait pas non plus à la culture de l'élite. Il était donc difficile de retrouver leur trace dans les archives. Mais leur interdisciplinarité m’a permis de recueillir quelques données. Sur les conseils d'un directeur des archives de Téhéran, j'ai élargi ma recherche aux documents concernant la prostitution sous le règne des Pahlavi. J'ai ainsi fini par la " danse de cabaret " classée dans une sous-catégorie réservée aux travailleuses du sexe appelées " servantes de la nuit" (khidmatguzaran-i-shab). La police urbaine forçait les travailleuses du sexe, ainsi que les femmes travaillant dans le milieu de la nuit, à quelque titre que ce soit (y compris les danseuses), à se soumettre au dépistage des maladies vénériennes. Ce règlement, dont j'avais appris l'existence par Naghmah et Rahilah, était la preuve choquante d'une double mesure appliquée dans le système législatif de la période prérévolutionnaire : d'un côté, la loi interdisait de faciliter les rapports sexuels dans l'espace public; de l'autre, le milieu de la nuit forçait ses employées à se faire dépister contre les maladies vénériennes tout en les exploitant par le biais du fishkhuri.

\section{Le nouveau spectre des danseuses de cabaret iraniennes et le regard porté par les universitaires}

La position subalterne occupée par la danseuse de cabaret dans le discours public a compliqué l'écriture raisonnée de son histoire, puisque la totalité des sources textuelles, visuelles ou filmées donnaient d'elle une image négative. J'ai procédé au croisement des informations recueillies dans les archives ou lors des interviews, tout en m'efforçant d'aiguiser ma sensibilité d'artiste et de chercheuse à la persistance des représentations biaisées des danseuses de cabaret. Le résultat de mes investigations concerne ainsi tout autant les préjugés les concernant que les danseuses elles-mêmes.

Voulant tester ces préjugés dans d'autres contextes, j'ai présenté certains aspects de mes recherches sur ces trois genres - danse nationale, mouvements rythmiques et danse de cabaret - lors de diverses rencontres universitaires consacrées à la danse, à l'Iran et au Moyen-Orient. Ces trois sujets ont été reçus de manière différente selon la familiarité culturelle et les choix disciplinaires des participants.

Dans les colloques consacrés à la danse, j'ai constaté un manque d'intérêt général pour les genres de danse de l'époque prérévolutionnaire. Domaine eurocentré de par ses origines, malgré l'orientation récente vers la théorie orientaliste et l'investigation postcoloniale, les études sur la danse restent foncièrement éloignées du contexte historique et culturel moyen-oriental. La danse nationale iranienne est donc souvent ramenée à un projet colonialiste, et l'on considère que ses créateurs locaux manquent d'autonomie, se contentant de plagier le vocabulaire des danses occidentales. La danseuse de cabaret iranienne, en revanche, est envisagée comme une danseuse du ventre qui s'orientalise, image qui s'accorde avec la vogue de la danse orientaliste en Occident, sans lien avec la

place socioculturelle qu'elle occupait dans l'Iran prérévolutionnaire. À l'inverse, le statut géopolitique de l'Iran, et plus particulièrement ses relations avec les États-Unis, a fait que les relations entre l'État islamique et la population, notamment la censure et la répression visant les femmes et l'expression artistique, sont au centre de l'attention des médias, 
du marché de l'art et des milieux universitaires occidentaux. Heather Rastovac-Akbarzadeh, qui a étudié l'état actuel du marché occidental concernant la danse iranienne, estime à ce propos qu'on porte sur elle un "regard de sauveur ${ }^{18}$ ". C'est peut-être pour cette raison que parmi les trois genres, c'est celui des harikat-i-mawzun - " mouvements rythmiques »-qui, dans le contexte sociopolitique plus connu qui est le sien, possède un aspect esthétique subversif, et est privilégié par les chercheurs s'intéressant à la danse.

Figure culturelle bien connue des cercles universitaires iraniens, la très controversée danseuse de cabaret, incarnation des mystères de la vie nocturne de Téhéran, suscitait la curiosité et l'enthousiasme. Ce n'était pas le cas de son homologue, la danseuse nationale, pratiquant un genre à l'esthétique moins bien connue, dans un cadre moins familier. On se demandait en outre si la danse était véritablement un sujet crédible et pertinent de recherche savante. Dans la hiérarchie des disciplines universitaires reconnues, la danse se trouvait en bas de l'échelle (elle l'est toujours), après la politique, l'économie, la littérature et les domaines plus récents tels que la sexualité dans les milieux de culture musulmane et les études de cinéma.

\section{L'avenir des études sur la danse en Iran}

Malgré les complications d'ordre logistique, sociétal et psychologique que présentaient mes sujets de recherche et les entretiens avec mes interlocutrices, et en dépit d'un périple relativement difficile, mais plaisant, à travers l'histoire de la danse iranienne, l'avenir de la danse et de son étude semble grandement s'éclaircir en Iran. La culture de la danse est florissante dans le pays (peut-être pas d'un point de vue esthétique), le terme danse a trouvé une nouvelle vie dans le discours officiel, et les chaînes de télévision satellite, ainsi que les réseaux sociaux, ont normalisé la danse (voire les tenues légères) aux yeux du spectateur iranien. Malgré les restrictions de l'ère post-révolutionnaire, les femmes artistes sont désormais acceptées par la société et jouissent même d'un certain prestige. La nostalgie récente pour l'ère des Pahlavi a contribué à faire naître une fascination pour l'histoire des arts et de la culture populaire. Plusieurs thèses de doctorat en anthropologie consacrées à la scène théâtrale et aux rituels iraniens sont actuellement menées sous la direction d'universitaires non iraniens.

Le travail des futurs historiens de la danse et des autres formes de culture populaire en Iran se heurtera cependant à la disparition de ceux et celles qui en ont été les acteurs ou les témoins. En l'espace de quelques mois, et malgré tous mes efforts, j'ai perdu le contact avec quatre des interlocutrices citées plus haut. En 2014, à la fin de mes études de doctorat, trois d'entre elles étaient décédées. Parmi les conséquences néfastes des soubresauts qu'a connus l'Iran au XXe siècle, avec ses bouleversements politiques, ses contestations idéologiques et ses préjugés socioculturels, il faut compter la perte des voix (subalternes) sans lesquelles notre connaissance de l'histoire reste partielle.

Cette contribution a été traduite

de l'anglais par Françoise Jaouën. 


\section{Ida Meftahi}

Ida Meftahi est professeure assistante en Études sur le Moyen-Orient (Boise State University), spécialiste de I'histoire de I'Iran moderne, avec un accent particulier porté sur les liens entre la politique, le genre et la performance. Son premier livre, Gender and Dance in Modern Iran: Biopolitics on Stage (Londres, Routledge, 2016), a reçu le prestigieux prix Latifeh Yarshater de I'Association for Iranian Studies (AIS), pour sa contribution originale au domaine des études sur les femmes iraniennes. Elle travaille actuellement à un second manuscrit, provisoirement intitulé Tulip Grove, Tehran: Urban Geopolitics, Gender, and Performative Culture in Modern Iran, qui se concentre sur la rue historique Lalehzar, à Téhéran, et ses environs, entre 1880 et 1960.

\section{NOTES}

1. Sur l'histoire de la danse en couple, voir Ida Meftahi, "Intimate Embraces with Ajnabi (inconnus): A Political History of Partner Dancing in Tehran, 1920-1950 ", dans Clio, Femmes, Genre, Histoire, vol. 46, n 2, 2018.

2. Sur l'Organisation nationale folklorique, voir le livre de mémoires du directeur artistique Robert de Warren, Destiny's Waltz: In Step with Giants, Cork, Publish on Demand Global LLC, 2012 ; sur I'histoire du ballet en Iran, voir Haideh Ahmadzadeh, fondatrice de la Compagnie nationale de ballet, My Life as a Persian Ballerina, s.I., Lulu, 2008. Voir également la contribution de Nima Kiann dans Saloumeh Gholami (dir.), Dance in Iran: Past and Present, Wiesbaden, Reichert Verlag Wiesbaen, 2016.

3. Voir le compte-rendu du concert de 1998 par Camelia Entekhabifard, "Behind the Veil », dans Mother Jones, mai-juin 2001 [en ligne, URL : www.motherjones.com/ politics/20].

4. J'ai initialement publié un article sur ce genre sous le pseudonyme de Zeinab Stellar : "From "Evil-Inciting" Dance to Chaste "Rythmic Movements": A Genealogy of Modern Islamic Dance Theater in Iran », dans Karin Van Nieuwkerk (dir.), Muslim Rap, Halal Soaps, and Revolutionary Theater: Artistic Developments in the Muslim Cultural Sphere, Austin, University of Texas Press, 2011, p. 231-256.

5. La salle Roudaki est, aujourd'hui encore, la plus prestigieuse salle de spectacle en Iran. Elle abritait l'Organisation nationale folklorique et la Compagnie nationale de ballet.

6. Sur les lieux, privés et publics, réservés à la pratique, la performance et l'éducation en matière de danse, voir Parmis Mozaffari, "Dance and the Borders of Public and Private Life in Post-Revolution Iran », dans Massoumeh Torfeh et Annabelle Sreverny-Mohammadi (dir.), Cultural Revolution in Iran: Contemporary Popular Culture in the Islamic Republic, Londres, I.B. Tauris, 2013.

7. L'ethnomusicologue Erum Naqvi a étudié l'usage créatif des traditions du mouvement par cette jeune génération dans le théâtre iranien contemporain ; voir Erum Naqvi, "Reinventing Ruhawzi », dans Middle East Journal of Culture and Communication, vol. 13, n ${ }^{\circ} 1$, mai 2020, p. 28-48.

8. Soheila Shahshahani, "Body as a Means of NonVerbal Communication in Iran ", dans S. Shahshahani (dir.), Body as Medium of Meaning, Münster, LIT, 2004, p. 57-71.

9. L'épisode 20 du podcast produit par Radio Marz et diffusé le 20 mai 2020 sur les réseaux sociaux cite le témoignage de plusieurs personnes de la génération postrévolutionnaire qui se sont senties mises à l'écart lorsque les gens ont commencé à danser lors de réunions amicales.

10. Anthony Shay, Choreophobia: Solo Improvised Dance in the Iranian World, Costa Mesa, Californie, Mazda (coll. «Bibliotheca Iranica, Performing Arts Series », 4), 1999.

11. Le chapitre VI de mon livre est consacré à une analyse critique du discours anticolonialiste et anti-obscénité dans les périodiques islamiques entre 1930 et 1979.

12. Ida Meftahi, "The Sounds and Moves of Ibtizal in 20th-Century Iran », dans International Journal of Middle East Studies, vol. 48, n 1, février 2016, p. 151-155.

13. Sasan Fatemi, ethnomusicologue pionnier établi en Iran, a beaucoup écrit sur les troupes mutribi. Voir G. J. Breyley et Sasan Fatemi, Iranian Music and Popular Entertainment: From Motrebi to Losānjelesi and Beyond, Abingdon, Routledge, 2016.

14. À plusieurs reprises, Rahilah marqua une nette distinction entre les cafés assez mal réputés (kafah) et les cabarets, à la clientèle plus familiale. Selon elle, les premiers étaient des endroits dangereux, sales et fréquentés seulement par un public plus agressif exclusivement masculin, et où les artistes étaient moins bien payées.

15. Elle me présenta comme une chercheuse écrivant I'histoire de Lalehzar. Je ne m'intéressais pas à l'époque à ce quartier, mais cette rencontre a orienté mon projet dans cette direction.

16. Kamran Talatoff, spécialiste de littérature, a retracé en détail la vie de l'actrice et danseuse de cabaret Kubra Sae'idi (née Shahrzad) dans Modernity, Sexuality, and Ideology in Iran: The Life and Legacy of a Popular Female Artist, Syracuse, New York, Syracuse University Press, 2011.

17. À la fin de notre entretien, Naghmah me montra sur le mur une photographie de son fils, installé en Europe depuis des décennies, en $\mathrm{m}^{\prime}$ implorant de ne pas nuire à sa réputation en menant mes recherches.

18. Heather Rastovac-Akbarzadeh, « Do Iranian Dancers Need Saving? Savior Spectatorship and the Production of Iranian Dancing Bodies as "Objects of Rescue" », dans Susan Manning, Janice Ross et Rebecca Schneider (dir.), The Future of Dance Studies, Madison, Wisconsin, University of Wisconsin Press, 2020, p. 453-470. 